\title{
Residual Symptoms, Lung Function and Imaging Findings in Patients Recovering from SARS-CoV-2 Infection
}

fares darawshy ( $\sim$ fares@hadassah.org.il)

Hadassah Hospital Ein Kerem: Hadassah Medical Center https://orcid.org/0000-0003-0177-908X Ayman Abu Rmeileh

Hadassah Hospital Ein Kerem: Hadassah Medical Center

Rottem Kuint

Hadassah Hospital Ein Kerem: Hadassah Medical Center

Dan Padawer

Hadassah Hospital Ein Kerem: Hadassah Medical Center

Khalil Karim

Hadassah Hospital Ein Kerem: Hadassah Medical Center

Zvi G Fridlender

Hadassah Hospital Ein Kerem: Hadassah Medical Center

Uri Laxer

Hadassah Hospital Ein Kerem: Hadassah Medical Center

Polina Cohen Goychman

Hadassah Hospital Ein Kerem: Hadassah Medical Center

Neville Berkman

Hadassah Hospital Ein Kerem: Hadassah Medical Center

\section{Research}

Keywords: COVID-19, post-COVID, dyspnea, pulmonary function, FVC

Posted Date: July 14th, 2021

DOl: https://doi.org/10.21203/rs.3.rs-685778/v1

License: (c) (i) This work is licensed under a Creative Commons Attribution 4.0 International License.

Read Full License 


\section{Abstract}

\section{Rationale}

Symptoms following acute COVID-19 infection are common, but their relationship to initial COVID-19 severity is unclear. We hypothesize that residual symptoms are related to disease severity, and severe acute COVID-19 infection is more likely to cause residual pulmonary damage. This study aims to evaluate symptoms, lung function and abnormal imaging within 3 months following COVID-19 infection, and whether they are related to initial disease severity.

\section{Methods}

A cross-sectional study was carried out at a designated post-COVID clinic in Hadassah Medical Center, Jerusalem, Israel. Patients with PCR-confirmed SARS-CoV-2 infection were evaluated within 12 weeks following infection and included both admitted and non-admitted subjects. All participants underwent assessment of symptoms, quality of life (SGRQ), pulmonary function tests, and imaging.

\section{$\underline{\text { Results }}$}

A total of 208 patients (age $49.3 \pm 16$ years) were included in the study. Initial disease severity was mild in 86 , moderate in 49 and severe in 73 patients. At the time of follow up, there were no differences in frequency of residual symptoms or in SGRQ score between groups. Patients with severe COVID-19 were more likely to have residual dyspnea $(p=0.04)$, lower oxygen saturation $(p<0.01)$, lower FVC and TLC $(p<0.001, p=0.03$ respectively), abnormal CXR $(p<0.01)$ and abnormal CT scan $(p<0.01)$ compared to other groups.

\section{Conclusion}

Frequency of symptoms and impairment of quality of life at 12 weeks follow up are common and are not related to severity of initial COVID-19 disease. In contrast, reduced lung function and abnormal pulmonary imaging are more common in patients with more severe acute COVID-19 infection.

\section{Background}

The severe acute respiratory syndrome coronavirus 2 (SARS-Cov-2) outbreak began in China in December $2019^{1,2}$. Since then, the virus has caused a global pandemic with over a hundred and fifty million persons infected and over 3 million deaths. The clinical spectrum of acute coronavirus induced disease (COVID-19) is wide, and ranges from asymptomatic infection, mild self-limiting disease, to acute lifethreatening respiratory failure ${ }^{2,3}$. Furthermore, the acute infection is not limited to the respiratory system, and may lead to multisystem involvement, including neurological, gastrointestinal, thromboembolic and cardiovascular disturbances ${ }^{4}$. 
In addition to the acute illness, long term effects of COVID-19 have also emerged, and patients may suffer from persistent symptoms, impaired lung function, and both pulmonary and extra-pulmonary complications ${ }^{5}$. The increasing number of patients suffering from long-term effects has led to the implementation of terms such as "long-COVID" or "post-COVID"5,6, in which patients have persistent multisystem symptoms and complications. Currently, post-COVID is defined as the presence of symptoms extending beyond 3 weeks of acute COVID-19 initial presentation, and post-COVID may even extend beyond 12 weeks ${ }^{6-8}$.

Previous reports have detailed residual symptoms following SARS-CoV-2 infection. Persistent symptoms include dyspnea, fatigue, chest pain and cognitive dysfunction, and were seen in $87.4 \%$ of recovered patients ${ }^{9}$. Dyspnea is the most common persistent symptom, present in $42-66 \%$ of patients at $60-100$ days of follow up 5,9,10. Additional studies have also shown persistent symptoms and functional impairment after COVID-19, particularly in hospitalized patients with severe disease, but also in those with mild disease ${ }^{5,11-15}$.

Chest imaging and lung function may also be impaired following SARS-CoV-2 infection. In 55 patients who recovered from COVID-19, abnormal chest computerized tomography (CT) scan was found in 39 of them, with impaired lung function in 14 patients ${ }^{16}$. Other studies evaluating radiological abnormalities showed that up to $56 \%$ of patients have persistent radiographic abnormalities after 12 weeks of follow up 17. In several cohorts that assessed lung function in patients who survived COVID-19 hospitalization, restrictive lung abnormality and diffusion limitation demonstrated in $22-38 \%$, and $24-71.7 \%$

18,19 respectively, especially in patients with severe COVID-19. In a large Chinese cohort that included 1733 patients, Huang et al. showed diffusion impairment at follow up in $56 \%$ in severely ill patients, and abnormal CT pattern in $50 \%$ of 349 patients at 6 months ${ }^{20}$.

There is little data analyzing the long-term residual effects in COVID-19 survivors in relation to initial disease severity. Most published studies in post-COVID-19 patients have focused on those with severe initial disease or in hospitalized patients. A recent study of 63 COVID-19 survivors compared both hospitalized and non-hospitalized patients and found persistent dyspnea and fatigue in both groups. Hospitalized patients had lower total lung and diffusion capacity ${ }^{21}$. Another study that evaluated mainly outpatients, showed that symptomatic patients have significantly reduced lung function, most notably impaired gas transfer ${ }^{22}$.

We hypothesize that residual symptoms in COVID-19 survivors are related to disease severity, and patients with severe disease are more likely to develop residual symptoms, and pulmonary damage as assessed by impaired lung function or abnormal imaging. This study aims to evaluate residual symptoms, lung function and imaging findings following SARS-CoV-2 infection.

\section{Methods}




\section{Study design and participants}

We conducted a cross-sectional cohort analysis which was carried out at the post COVID clinic in Hadassah Medical Center, Jerusalem, Israel. The study protocol was approved by the ethics committee of Hadassah - Hebrew University Medical Center (number HMO-0294-20) and informed consent was obtained from all participants in the study. Post COVID-19 patients were invited to the clinic by direct phone calls following hospital discharge from COVID wards, addition of written instructions in hospital discharge summaries as to post-COVID clinic details and advertisement in social media targeting outpatients or those who were admitted in other hospitals. After their presentation to the clinic, participants were asked to participate in the study by a research physician.

Between August 2020 and February 2021, we recruited participants who presented to the post-COVID outpatient clinic. Patients were $>18$ years of age and diagnosed with SARS-CoV-2 infection by RT-PCR, on average of 12 weeks before enrolment. We included those who were admitted and managed as inpatients, and outpatients managed at home.

\section{Clinical assessment}

All data was obtained during the clinic visit. Routine demographic information was collected, as well as other clinical information including date of COVID-19 diagnosis, comorbidities, smoking history, symptoms and clinical features of acute COVID-19. Inpatient data was extracted through review of patient's electronic medical records. Data relating to acute COVID19 included peak oxygen requirements, oxygen saturation at presentation (if available), chest x-ray findings and treatment during admission. Imaging during admission reported as normal or abnormal. All patients undergone clinical assessment regrading residual symptoms, and oxygen saturation both at rest and exercise.

Participants were divided into three major groups of initial COVID-19 severity, according to the national health institute COVID-19 treatment guidelines ${ }^{3}$ : mild, moderate and severe. In mild disease individuals have any of the various signs and symptoms of COVID-19, but without shortness of breath or abnormal chest imaging. In moderate disease individuals show evidence of lower respiratory disease during clinical assessment or imaging and have oxygen saturation of $94 \%$ or more on room air at sea level. In severe disease, individuals have oxygen saturation less than $94 \%$ on room air, respiratory frequency $>30$ breaths/min, or lung infiltrates $>50 \%$. In addition, patients were compared as inpatients and outpatients' groups.

During post-COVID clinic assessment, all participants were asked to fill out Saint George respiratory questionnaire (SGRQ) for measurement of impact on overall health, daily life, and perceived well-being 23,24 .

All participants were asked to perform a spirometry and CXR during their visit. Further investigation was performed according to initial disease severity. No further investigation was performed in participants 
with mild disease. Chest CT, body plethysmography, and diffusing capacity of carbon monoxide (DLCO), were performed in those with severe disease or according to physician judgment.

Spirometry and pulmonary function tests were performed according to the American Thoracic Society/European Respiratory Society standards ${ }^{25,26}$. Data included forced expiratory volume at 1 second (FEV1), forced vital capacity (FVC), the ratio between the two parameters: FEV1/FVC, static lung volumes, and DLCO using the single breath technique. All data presented as percentage of predicted normal values.

\section{Data analysis}

The data were analyzed using SPSS software version 25. Statistical significance was considered for $\mathrm{p}$ value lower than 0.05 .

Descriptive statistics were performed using means, standard deviations and ranges for the continuous variables, and frequencies for the discrete variables. Differences between patient groups (according to initial COVID-19 severity) were assessed using Chi-square tests for the discrete variables, and MannWhitney and Kruskal-Wallis tests for the continuous variables. Post hoc analyses were conducted using Bonferroni corrections for multiple comparisons. To analyze the impact of clinical parameters on persistent symptoms and respiratory outcomes, we used a multivariate analysis which were conducted using Logistic regressions. Separate Logistic regressions were conducted for assessing the probability to have persistent symptoms, reduced FVC, abnormal CXR, and abnormal CT at the time of study participation.

\section{Results}

\section{Baseline Characteristics}

A total of 208 patients (age $49.3 \pm 16$ years) were included with a mean follow-up of $80 \pm 50$ days after acute COVID-19 infection (Table 1). $56.3 \%$ of patients were male and $43.7 \%$ were female. Hypertension (n $=41,19.7 \%)$ and hyperlipidemia $(n=40,19.2 \%)$ were the most frequent reported comorbidities. Most of the patients were non-smokers $(n=174,83.7 \%), 7.2 \%$ were current smokers and $9.1 \%$ were past smokers. Patients with severe COVID-19 were older and had more comorbidities compared to those with mild and moderate disease. 
Table 1

Baseline patients clinical characteristics by initial disease severity.

$N(\%)$ or Mean \pm SD

p-value

\begin{tabular}{|c|c|c|c|c|c|}
\hline & $\begin{array}{l}\text { Total } \\
(n=208)\end{array}$ & $\begin{array}{l}\text { Mild } \\
(n=86)\end{array}$ & $\begin{array}{l}\text { Moderate } \\
(n=49)\end{array}$ & $\begin{array}{l}\text { Severe } \\
(n=73)\end{array}$ & \\
\hline Age, years & $49.3 \pm 16.1$ & $42.3 \pm 15.2$ & $48.5 \pm 15.9$ & $58 \pm 13.1$ & $<0.001$ \\
\hline Male & $117(56.3)$ & $38(44.2)$ & $29(59.2)$ & $50(68.5)$ & 0.007 \\
\hline Female & $91(43.8)$ & $48(55.8)$ & $20(40.8)$ & $23(31.5)$ & \\
\hline Comorbidities & 41 (19.7) & $10(11.6)$ & $7(14.3)$ & $24(32.9)$ & $<0.001$ \\
\hline Hypertension & $29(13.9)$ & $7(8.1)$ & $6(12.2)$ & $16(21.9)$ & 0.04 \\
\hline Diabetes mellitus & 40 (19.2) & $8(9.3)$ & $6(12.2)$ & $26(35.6)$ & $<0.001$ \\
\hline Hyperlipidemia & $12(5.8)$ & $3(3.5)$ & $0(0)$ & $9(12.3)$ & - \\
\hline IHD & $16(7.7)$ & $5(5.8)$ & $4(8.2)$ & $7(9.6)$ & 0.67 \\
\hline Cancer & $10(4.8)$ & $4(4.6)$ & $3(6.1)$ & $3(4.1)$ & 0.56 \\
\hline Asthma/COPD & $3(1.4)$ & $0(0)$ & - & $3(4.1)$ & - \\
\hline AF & $3(1.4)$ & $1(1.1)$ & - & $2(2.7)$ & - \\
\hline Heart failure & $1(0.5)$ & - & - & $1(1.4)$ & - \\
\hline Cirrhosis & $46(22.1)$ & $15(17.4)$ & $11(12.8)$ & $20(23.3)$ & 0.32 \\
\hline \multicolumn{6}{|l|}{ Other } \\
\hline Smoking & $174(83.7)$ & $69(80.2)$ & $44(89.8)$ & $61(83.6)$ & 0.35 \\
\hline Never smoker & $15(7.2)$ & $9(10.5)$ & $4(8.1)$ & $2(2.7)$ & \\
\hline Active smoker & $19(9.1)$ & $8(9.3)$ & $1(2.1)$ & $10(13.7)$ & \\
\hline \multicolumn{6}{|l|}{ Past smoker } \\
\hline COVID-19 illness & 147 (70.7) & $48(55.8)$ & 37 (75.5) & $62(84.7)$ & $<0.001$ \\
\hline Cough & $88(42.3)$ & $7(8.1)$ & $29(59.2)$ & $52(71.2)$ & $<0.001$ \\
\hline Dyspnea & $92.2 \pm 6.09$ & $96.5 \pm 1.7$ & $95.2 \pm 3$ & $88 \pm 6.1$ & $<0.001$ \\
\hline Sao2 & & & & & \\
\hline
\end{tabular}

IHD: Ischemic heart disease, COPD: Chronic obstructive pulmonary disease, SaO2: Oxygen saturation at room air, HFNC: High Flow Nasal Cannula, CT: computerized tomography, Other therapy include convalescent plasma, anti-IL-6, lopinavir-ritonavir, and hydroxychloroquine 


\begin{tabular}{|c|c|c|c|c|c|}
\hline Oxygen requirements & $142(68.3)$ & $86(100)$ & $43(87.7)$ & $13(17.8)$ & $<0.001$ \\
\hline No oxygen & $43(20.7)$ & - & $6(12.3)$ & $37(50.7)$ & $<0.001$ \\
\hline Supplemental oxygen & $14(6.7)$ & - & - & $14(19.2)$ & $<0.001$ \\
\hline HFNC & $9(4.3)$ & - & - & $9(12.3)$ & $<0.001$ \\
\hline \multicolumn{6}{|l|}{ Mechanical ventilation } \\
\hline Chest X-Ray & $92(44.2)$ & $66(76.7)$ & $16(32.7)$ & $10(13.7)$ & \multirow[t]{3}{*}{$<0.001$} \\
\hline Not performed & $38(18.3)$ & $20(23.3)$ & $12(24.5)$ & $6(8.2)$ & \\
\hline Normal & 78 (37.5) & - & $21(42.8)$ & $57(78.1)$ & \\
\hline \multicolumn{6}{|l|}{ Abnormal } \\
\hline Chest CT & $177(85.1)$ & 86 & $44(89.9)$ & $47(64.4)$ & \multirow[t]{3}{*}{$<0.001$} \\
\hline Not performed & - & - & - & - & \\
\hline Normal & $31(14.9)$ & - & $5(10.1)$ & $26(35.6)$ & \\
\hline \multicolumn{6}{|l|}{ Abnormal } \\
\hline Therapy (at infection) & $27(13)$ & $1(1.2)$ & $3(6.1)$ & $23(31.5)$ & $<0.001$ \\
\hline Antibiotics & $41(19.7)$ & - & - & $41(56.2)$ & $<0.001$ \\
\hline Remdesevir & $61(29.3)$ & $1(1.2)$ & $5(10.2)$ & $55(75.3)$ & $<0.001$ \\
\hline Dexamethasone & 67 (32.3) & $2(2.3)$ & $9(18.8)$ & $56(76.6)$ & $<0.001$ \\
\hline Anticoagulation & $22(10.5)$ & - & $3(6.1)$ & 10 (13.7) & $<0.001$ \\
\hline \multicolumn{6}{|l|}{ Other } \\
\hline Length of stay, days & $8.92 \pm 9.64$ & $2.7 \pm 2.8$ & $3.9 \pm 3.6$ & $13.5 \pm 10.8$ & $<0.001$ \\
\hline
\end{tabular}

During acute COVID-19 illness, $70.7 \%$ of patients had cough, $42.3 \%$ had dyspnea, and $89.4 \%$ had general weakness. Patients in the severe group had lower oxygen saturation on room air at presentation (Table 1 ). $68.3 \%$ of the patients didn't need respiratory support, and only $4.3 \%$ were mechanically ventilated, all in the severe group. $20.7 \%$ were treated with supplemental oxygen and $6.7 \%$ required high flow nasal cannula.

116 (55.8\%) patients underwent CXR on presentation, which was abnormal in 78 (37.5\%). CXR was not performed in patients with mild disease, and most of those who underwent CXR were in the severe group. 
Similarly, chest CT was performed in 31 (14.9\%) patients and was abnormal in all cases. Therapy during admission and length of stay is presented in Table 1.

\section{Follow Up Visit}

Mean follow up period was $81 \pm 50$ days, without significant difference between the three groups. $94.2 \%$ patients had at least one residual symptom, and only $5.8 \%(n=12)$ of the patients were asymptomatic. There were no significant differences in the frequency of pulmonary or extrapulmonary symptoms between patients with different initial disease severity, (Table 2). The most common symptom at follow up was fatigue [ $61.6 \%, 49 \%, 67.1 \%$ in mild, moderate and severe COVID-19, respectively, $(p=0.13)$ ] (Fig. 1a). Only dyspnea was more common in patients with severe vs. mild and moderate COVID-19 [68.5\% vs $48.8 \%$ and $57.1 \%$, $(p=0.04)]$. Other residual symptoms and their frequencies are reported in Table 2 and Fig. 1. Quality of life as measured by SGRQ score, was significantly reduced in all patients, without significant difference between mild, moderate or severe COVID-19 survivors. Patients with severe disease has significantly lower oxygen saturation compared to patients with mild and moderate disease, both at rest $(95.3 \pm 2.5$ vs $96.8 \pm 1.5$, and $96.4 \pm 1.5, p<0.001)$ and exercise $(93.3 \pm 4$ vs. $96.2 \pm 1.7$ and $95.8 \pm 1.7, p<0.001)$. 
Table 2

Residual symptoms, SGRQ score, lung function and imaging findings on follow up, by initial disease severity.

\begin{tabular}{|c|c|c|c|c|c|}
\hline & \multicolumn{4}{|c|}{$N(\%)$ or Mean \pm SD } & \multirow[t]{2}{*}{$\mathrm{p}$-Value } \\
\hline & $\begin{array}{l}\text { Total } \\
(n=208)\end{array}$ & $\begin{array}{l}\text { Mild } \\
(n=86)\end{array}$ & $\begin{array}{l}\text { Moderate } \\
(n=49)\end{array}$ & $\begin{array}{l}\text { Severe } \\
(n=73)\end{array}$ & \\
\hline Follow-up, days & $81 \pm 50$ & $85 \pm 55$ & $80 \pm 48$ & $74 \pm 45$ & 0.48 \\
\hline Residual symptoms & $12(5.8)$ & $3(3)$ & $7(14.2)$ & $2(2)$ & 0.52 \\
\hline Asymptomatic & $40(9.2)$ & $13(15.7)$ & $9(21.4)$ & $18(25.4)$ & \\
\hline Pulmonary & $116(55.8)$ & $20(24.1)$ & $9(21.4)$ & $11(15.5)$ & \\
\hline Extrapulmonary & $120(57.7)$ & $50(60.2)$ & $24(57.1)$ & $42(59.2)$ & \\
\hline \multicolumn{6}{|l|}{ Both } \\
\hline Dyspnea & $120(57.7)$ & $42(48.8)$ & $28(57.1)$ & $50(68.5)$ & .04 \\
\hline Cough & $47(22.6)$ & $17(19.8)$ & $16(32.7)$ & $14(19.2)$ & 0.16 \\
\hline Exercise Intolerance & $50(24)$ & $18(20.9)$ & $13(26.5)$ & $19(26.0)$ & 0.68 \\
\hline Chest Pain & $34(16.3)$ & $14(16.3)$ & $7(14.3)$ & $13(17.8)$ & 0.87 \\
\hline Fatigue & $126(60.6)$ & $53(61.6)$ & $24(49.0)$ & $49(67.1)$ & 0.13 \\
\hline Arthralgia / Myalgia & $45(21.6)$ & $22(25.6)$ & $7(14.3)$ & $16(21.9)$ & 0.31 \\
\hline Hair Loss & $28(13.5)$ & $15(17.4)$ & $6(12.2)$ & $7(9.6)$ & 0.34 \\
\hline Anxiety & $20(9.6)$ & $11(12.8)$ & $4(8.2)$ & $5(6.8)$ & 0.41 \\
\hline Cognitive Dysfunction & $59(28.4)$ & $28(32.6)$ & $12(24.5)$ & $19(26.0)$ & 0.52 \\
\hline Anosmia / Ageusia & $16(7.7)$ & $10(11.6)$ & $3(6)$ & $3(4)$ & 0.18 \\
\hline SGRQ score & $39.6 \pm 25.6$ & $37 \pm 24.8$ & $42.8 \pm 25.6$ & $40.9 \pm 26.7$ & 0.67 \\
\hline $\mathrm{SaO} 2$ & $96.2 \pm 2.05$ & $96.8 \pm 1.5$ & $96.4 \pm 1.5$ & $95.3 \pm 2.5$ & $<0.001$ \\
\hline Rest & $95.0 \pm 3.0$ & $96.2 \pm 1.7$ & $95.8 \pm 1.7$ & $93.3 \pm 4.0$ & $<0.001$ \\
\hline \multicolumn{6}{|l|}{ Exercise } \\
\hline \multicolumn{6}{|l|}{ Spirometry } \\
\hline FEV1, \% predicted & $91.1 \pm 17.8$ & $94.7 \pm 16.2$ & $94.3 \pm 15$ & $85.2 \pm 19.7$ & $<0.001$ \\
\hline $\begin{array}{l}\text { Pulmonary symptoms } \\
\text { George Respiratory Que } \\
\text { volume at } 1 \text { second, FV } \\
\text { diffusion capacity for }\end{array}$ & $\begin{array}{l}\text { de dyspnea, } \\
\text { anaire, SaO2 } \\
\text { orced vital c } \\
\text { n monoxide }\end{array}$ & $\begin{array}{l}\text { gh, chest pai } \\
\text { ygen saturat } \\
\text { ity, RV: Resic } \\
\text { 2: chest X-ra }\end{array}$ & $\begin{array}{l}\text { dd exercise ir } \\
\text { at room air, } \mathrm{F} \\
\text { volume, TLC } \\
\text { : computeriz }\end{array}$ & $\begin{array}{l}\text { rance; SGRQ: } \\
\text { Forced expir } \\
\text { al lung capac } \\
\text { omography. }\end{array}$ & $\begin{array}{l}\text { aint } \\
\text { ory } \\
\text {, DLCO: }\end{array}$ \\
\hline
\end{tabular}




\begin{tabular}{|c|c|c|c|c|c|}
\hline FVC, \% predicted & $89.0 \pm 18.3$ & $94.9 \pm 14.8$ & $91.44 \pm 14.7$ & $81.3 \pm 20.8$ & $<0.001$ \\
\hline FEV1/FVC, \% predicted & $82 \pm 18.3$ & $81 \pm 11$ & $83 \pm 8$ & $82 \pm 10$ & 0.87 \\
\hline \multicolumn{6}{|l|}{ Lung volumes } \\
\hline RV, \% predicted & $98.2 \pm 38.6$ & $117.8 \pm 61.8$ & $104.2 \pm 31$ & $87.7 \pm 24$ & 0.13 \\
\hline TLC, \% predicted & $91.5 \pm 16.3$ & $96.4 \pm 11.1$ & $99.4 \pm 16.7$ & $86.3 \pm 16.4$ & 0.03 \\
\hline RV/TLC, \% predicted & $101.6 \pm 34.1$ & $119.2 \pm 59.2$ & $101.1 \pm 22.8$ & $94.5 \pm 19.6$ & 0.60 \\
\hline \multicolumn{6}{|l|}{ Diffusing capacity } \\
\hline DLCO, \% predicted & $84.2 \pm 14.5$ & $89.5 \pm 15.9$ & $86 \pm 15.1$ & $81.1 \pm 17.0$ & 0.28 \\
\hline CXR & $19(9.2)$ & $8(9.3)$ & 9 (18.3) & $2(2.7)$ & $<0.001$ \\
\hline Not performed & $130(62.8)$ & $68(79)$ & $32(65.3)$ & $30(41.1)$ & \\
\hline Normal & $58(28)$ & $10(11.7)$ & $8(16.3)$ & $41(56.2)$ & \\
\hline \multicolumn{6}{|l|}{ Abnormal } \\
\hline CT scan & $141(67.8)$ & $74(86)$ & $35(71.4)$ & $32(43.8)$ & 0.26 \\
\hline Not performed & $28(13.5)$ & $6(7)$ & $8(16.3)$ & $14(19.2)$ & \\
\hline Normal & $39(18.8)$ & $6(7)$ & $6(12.2)$ & $27(37)$ & \\
\hline \multicolumn{6}{|l|}{ Abnormal } \\
\hline Abnormal CT findings & $39(18.8)$ & $6(7)$ & $6(12.2)$ & $27(37)$ & $<0.001$ \\
\hline $\begin{array}{l}\text { Pulmonary symptoms i } \\
\text { George Respiratory Que } \\
\text { volume at } 1 \text { second, FV } \\
\text { diffusion capacity for } \mathrm{C}\end{array}$ & $\begin{array}{l}\text { ude dyspnea, } \\
\text { onnaire, SaO2: } \\
\text { Eorced vital ca| } \\
\text { on monoxide, }\end{array}$ & $\begin{array}{l}\text { Igh, chest pain } \\
\text { kygen saturatic } \\
\text { city, RV: Residu } \\
\text { R: chest X-ray, }\end{array}$ & $\begin{array}{l}\text { רd exercise int } \\
\text { at room air, FE } \\
\text { volume, TLC: } \\
\text { : computerize }\end{array}$ & $\begin{array}{l}\text { ance; SGRQ: } \\
\text { : Forced expi } \\
\text { tal lung capac } \\
\text { omography. }\end{array}$ & $\begin{array}{l}\text { aint } \\
\text { ory } \\
\text { y, DLCO: }\end{array}$ \\
\hline
\end{tabular}

\section{Lung function and imaging findings}

At follow up, spirometry was performed in total of 183 patients: 73,41 and 68 patients in the mild, moderate and severe group, respectively. Mean FVC (\% predicted) at follow up was $89 \pm 18$.3. Patients in the severe group has significantly lower FEV1 and FVC (Table 2, Fig. 1b). Absolute lung volumes and diffusion capacity were available in 62 patients: 14 in the mild, 14 in the moderate and 33 in the severe group. TLC was significantly reduced in the severe group compared to mild and moderate group $(86.3 \pm 16.4$ vs $96.2 \pm 11.1$ and $99.4 \pm 16.7, p=0.03$ ). DLCO was reduced in the severe group compared to other groups, although not statistically significant.

Patients in the severe group had more abnormal CXR at follow up compared to mild and moderate groups $(56.2 \%$ vs. $11.7 \%$ and $16.3 \%, p<0.001)$. There was no significant difference in CT findings 
between the three groups (Table 2). When comparing abnormal CT findings only, patients in the severe group had significantly higher abnormal CT imaging (Table 2). The pattern of injury seen on CT scan was residual ground glass opacities in 32 patients, reticular and interstitial abnormalities in 3 patients. One patient had pulmonary embolism. The remaining 3 patients had either incidental emphysema or mosaic pattern of attenuation.

\section{Patients requiring admission vs outpatients}

We performed a comparison between inpatients and outpatients (Table 3 ). About half of the patients were hospitalized $(55.8 \%)$, for an average of 8.92 days $(S D=9.64)$. Inpatients were older, had more comorbidities, and were more symptomatic during acute COVID-19 illness. There was a significant difference in in oxygen requirement $(51.8 \%$ of inpatients, $6.6 \%$ of outpatients, $p<0.001)$. In addition, inpatients were more likely to have abnormal CXR ( $65.5 \%$ vs $2.2 \%, p=0.09)$, abnormal CT scan $(26.7 \%$ vs $0, p<0.001$ ), and received therapy during their acute illness (Table 3). On follow up visit, there was no significant difference in residual symptoms or SGRQ score between outpatients and inpatients. Inpatients had significantly decreased SaO2, both at rest $(95.7 \pm 2.2$ vs $96.7 \pm 1.6$ in outpatients, $p<0.001)$ and exercise $(94.3 \pm 3.5$ vs. $96 \pm 2$ in outpatients, $p<0.001)$. The following lung functions parameters were significantly reduced in inpatients vs outpatients: FEV1, FVC and TLC (Table 3, Fig. 2b). DLCO was also lower in inpatients vs outpatients, but without statistical significance (81.2 \pm 16.3 vs. $90.2 \pm 15.3, p=$ 0.28). Inpatients had more abnormal CXR at follow up compared to outpatients ( $37 \%$ vs $16.3 \%, p<0.001$ ), and more abnormal CT scan $(28.5 \%$ vs $6.5 \%, \mathrm{p}<0.01)$. 
Table 3

comparison of outpatients Vs inpatients. Major Baseline clinical characteristics, residual symptoms, SGRQ score, lung function and imaging findings at follow up.

\begin{tabular}{|c|c|c|c|}
\hline & \multicolumn{2}{|c|}{$N(\%)$ or Mean \pm SD } & \multirow{2}{*}{$\begin{array}{l}\text { p-Value } \\
\text { p-value }\end{array}$} \\
\hline & Outpatients & Inpatients & \\
\hline & $N=92$ & $N=116$ & \\
\hline Age, y & $44.8 \pm 115.8$ & $52.8 \pm 15.6$ & $<0.001$ \\
\hline Male & $50(54.3)$ & $67(57.7)$ & 0.62 \\
\hline Female & $42(45.7)$ & $49(42.3)$ & \\
\hline Hypertension & $13(14.1)$ & $28(24.1)$ & 0.07 \\
\hline Diabetes mellitus & $7(7.6)$ & $22(19)$ & 0.02 \\
\hline Hyperlipidemia & $9(9.8)$ & $31(26.7)$ & $<0.001$ \\
\hline Cancer & $4(4.3)$ & $12(10.3)$ & 0.11 \\
\hline Current / past smoking & $18(19.6)$ & $16(13.8)$ & 0.26 \\
\hline COVID-19 illness & $55(59.8)$ & $92(79.3)$ & $<0.001$ \\
\hline Cough & $26(28.3)$ & $62(53.4)$ & $<0.001$ \\
\hline Dyspnea & $83(90.2)$ & $103(88.8)$ & 0.74 \\
\hline Fatigue / Weakness & $91.7 \pm 5.7$ & $92.3 \pm 6.2$ & 0.31 \\
\hline \multicolumn{4}{|l|}{ Sao2 } \\
\hline Oxygen requirements & $86(93.4)$ & $56(48.2)$ & $<0.001$ \\
\hline No oxygen & $6(6.6)$ & 37 (31.8) & \\
\hline Supplemental oxygen & $0(0)$ & $14(12)$ & \\
\hline HFNC & $0(0)$ & $9(8)$ & \\
\hline Mechanical ventilation & & & \\
\hline
\end{tabular}

IHD: Ischemic heart disease, Sa02: Oxygen saturation at room air, HFNC: High Flow Nasal Cannula, CT: computerized tomography, Other therapy include convalescent plasma, anti-IL-6, lopinavirritonavir, and hydroxychloroquine, Pulmonary symptoms include: dyspnea, cough, chest pain, and exercise intolerance, SGRQ: Saint George Respiratory Questionnaire, SaO2: Oxygen saturation at room air, FEV1: Forced expiratory volume at 1 second, FVC: Forced vital capacity, RV: Residual volume, TLC: Total lung capacity, DLCO: diffusion capacity for carbon monoxide, CXR: chest X-ray, CT: computerized tomography 


\begin{tabular}{|c|c|c|c|}
\hline \multirow[b]{2}{*}{ CXR } & \multicolumn{2}{|c|}{$N(\%)$ or Mean \pm SD } & \multirow{2}{*}{$\begin{array}{l}\text { p-Value } \\
0.09\end{array}$} \\
\hline & $86(93.5)$ & $6(5.2)$ & \\
\hline Not performed & $4(4.3)$ & $34(29.3)$ & \\
\hline Normal & $2(2.2)$ & $76(65.5)$ & \\
\hline \multicolumn{4}{|l|}{ Abnormal } \\
\hline CT & $92(100)$ & 85 (73.3) & $<0.001$ \\
\hline Not performed & 0 & $0(0)$ & \\
\hline Normal & 0 & $31(26.7)$ & \\
\hline \multicolumn{4}{|l|}{ Abnormal } \\
\hline Therapy (at infection) & $2(2.2)$ & $25(21.6)$ & $<0.001$ \\
\hline Antibiotics & 0 & $41(35.3)$ & \\
\hline Remdesevir & $2(2.2)$ & $59(50.9)$ & \\
\hline Dexamethasone & $1(1.1)$ & $66(57.4)$ & \\
\hline Anticoagulation & $1(1.1)$ & $12(10.3)$ & \\
\hline \multicolumn{4}{|l|}{ Other } \\
\hline Length of stay & 0 & $9 \pm 9.6$ & - \\
\hline \multicolumn{4}{|l|}{ Follow up visit } \\
\hline Follow-up, days & $77.6 \pm 50$ & $82.2 \pm 50$ & 0.48 \\
\hline Residual symptoms & $1(1)$ & $11(9.5)$ & 0.8 \\
\hline Asymptomatic & $17(18.4)$ & $23(19.8)$ & \\
\hline Pulmonary & $20(21.7)$ & $20(17.2)$ & \\
\hline Extrapulmonary & $54(58.6)$ & $62(53.4)$ & \\
\hline \multicolumn{4}{|l|}{ Both } \\
\hline Dyspnea & $51(55.4)$ & $69(59.5)$ & 0.56 \\
\hline Cough & $23(25.0)$ & $24(20.7)$ & 0.46 \\
\hline
\end{tabular}

IHD: Ischemic heart disease, SaO2: Oxygen saturation at room air, HFNC: High Flow Nasal Cannula, CT: computerized tomography, Other therapy include convalescent plasma, anti-IL-6, lopinavirritonavir, and hydroxychloroquine, Pulmonary symptoms include: dyspnea, cough, chest pain, and exercise intolerance, SGRQ: Saint George Respiratory Questionnaire, Sa02: Oxygen saturation at room air, FEV1: Forced expiratory volume at 1 second, FVC: Forced vital capacity, RV: Residual volume, TLC: Total lung capacity, DLCO: diffusion capacity for carbon monoxide, CXR: chest X-ray, CT: computerized tomography 


\begin{tabular}{|c|c|c|c|}
\hline & $N(\%)$ or Mea & & $\mathrm{p}$-Value \\
\hline Exercise Intolerance & $25(27.2)$ & $25(21.6)$ & 0.35 \\
\hline Chest Pain & $17(18.5)$ & $17(14.7)$ & 0.46 \\
\hline Fatigue / Weakness & $59(64.1)$ & $67(57.8)$ & 0.35 \\
\hline Arthralgia / Myalgia & $25(27.2)$ & $20(17.2)$ & 0.08 \\
\hline Hair Loss & $15(16.3)$ & $13(11.2)$ & 0.28 \\
\hline Anxiety & $10(10.9)$ & $10(8.6)$ & 0.58 \\
\hline Cognitive Dysfunction & $29(31.5)$ & $30(25.9)$ & 0.37 \\
\hline Anosmia / Ageusia & $12(13)$ & $4(3.4)$ & 0.009 \\
\hline SGRQ & $40 \pm 25.7$ & $39.2 \pm 25.7$ & 0.67 \\
\hline $\mathrm{SaO} 2$ & $96.7 \pm 1.6$ & $95.7 \pm 2.2$ & $<0.001$ \\
\hline Rest & $96 \pm 2$ & $94.3 \pm 3.5$ & $<0.001$ \\
\hline \multicolumn{4}{|l|}{ Exercise } \\
\hline FEV1, \% predicted & $94.2 \pm 16.7$ & $88.1 \pm 18.4$ & $<0.001$ \\
\hline FVC, \% predicted & $94 \pm 15.4$ & $84.2 \pm 19.5$ & $<0.001$ \\
\hline FEV1/FVC, \% predicted & $81 \pm 11$ & $83 \pm 10$ & 0.87 \\
\hline $\mathrm{RV}, \%$ predicted & $114.5 \pm 50.9$ & $90.5 \pm 29$ & 0.13 \\
\hline TLC, \% predicted & $101 \pm 9$ & $87 \pm 17.3$ & 0.03 \\
\hline RV/TLC, \% predicted & $113 \pm 50$ & $96 \pm 22$ & 0.60 \\
\hline DLCO, \% predicted & $90.2 \pm 15.3$ & $81.2 \pm 16.3$ & 0.28 \\
\hline CXR & $6(6.5)$ & $14(12)$ & $<0.001$ \\
\hline Not performed & $71(77.2)$ & $59(51)$ & \\
\hline Normal & 15 (16.3) & 43 (37) & \\
\hline Abnormal & & & \\
\hline
\end{tabular}

IHD: Ischemic heart disease, Sa02: Oxygen saturation at room air, HFNC: High Flow Nasal Cannula, CT: computerized tomography, Other therapy include convalescent plasma, anti-IL-6, lopinavirritonavir, and hydroxychloroquine, Pulmonary symptoms include: dyspnea, cough, chest pain, and exercise intolerance, SGRQ: Saint George Respiratory Questionnaire, Sa02: Oxygen saturation at room air, FEV1: Forced expiratory volume at 1 second, FVC: Forced vital capacity, RV: Residual volume, TLC: Total lung capacity, DLCO: diffusion capacity for carbon monoxide, CXR: chest X-ray, CT: computerized tomography 


\begin{tabular}{|c|c|c|c|}
\hline & \multicolumn{2}{|c|}{ N (\%) or Mean \pm SD } & \multirow{2}{*}{$\begin{array}{l}\text { p-Value } \\
0.19\end{array}$} \\
\hline CT & $78(84.9)$ & $63(54.3)$ & \\
\hline Not performed & $8(8.6)$ & $20(17.2)$ & \\
\hline Normal & $6(6.5)$ & $33(28.5)$ & \\
\hline \multicolumn{4}{|l|}{ Abnormal } \\
\hline Abnormal CT findings & $6(6.5)$ & $33(28.5)$ & $<0.001$ \\
\hline \multicolumn{4}{|c|}{$\begin{array}{l}\text { IHD: Ischemic heart disease, SaO2: Oxygen saturation at room air, HFNC: High Flow Nasal Cannula, } \\
\text { CT: computerized tomography, Other therapy include convalescent plasma, anti-IL-6, lopinavir- } \\
\text { ritonavir, and hydroxychloroquine, Pulmonary symptoms include: dyspnea, cough, chest pain, and } \\
\text { exercise intolerance, SGRQ: Saint George Respiratory Questionnaire, SaO2: Oxygen saturation at room } \\
\text { air, FEV1: Forced expiratory volume at } 1 \text { second, FVC: Forced vital capacity, RV: Residual volume, TLC: } \\
\text { Total lung capacity, DLCO: diffusion capacity for carbon monoxide, CXR: chest X-ray, CT: } \\
\text { computerized tomography }\end{array}$} \\
\hline
\end{tabular}

\section{Predictors of respiratory outcomes}

The results are presented in Table 4. The regression model for predicting residual symptoms shows that age, $\mathrm{SaO} 2$ during acute COVID-19, smoking, and treatment with remdesivir and anticoagulation are associated with an increased probability of having persistent symptoms. 
Table 4

Odds ratio and confidence intervals for predictors of residual symptoms, reduced FVC, abnormal CXR and CT findings.

\begin{tabular}{|c|c|c|c|c|}
\hline & Residual Symptoms & Reduced FVC & Abnormal CXR & Abnormal CT \\
\hline \multirow[t]{2}{*}{ Age } & $0.95^{\star \star}$ & 1.03 & $1.11^{\star \star}$ & $1.11^{\star \star}$ \\
\hline & {$[0.91,0.99]$} & {$[0.99,1.07]$} & {$[1.05,1.18]$} & {$[1.04,1.18]$} \\
\hline \multirow[t]{2}{*}{ Gender (Male) } & 1.62 & 1.08 & $6.12^{\star}$ & 2.52 \\
\hline & [0.54. 4.84] & {$[0.35,3.31]$} & {$[1.36,27.57]$} & {$[0.58,10.92]$} \\
\hline \multirow[t]{2}{*}{ Any Medical history } & 3.08 & 1.24 & 0.63 & 0.53 \\
\hline & {$[0.93,10.20]$} & {$[0.35,4.33]$} & {$[0.14,2.72]$} & {$[0.12,2.35]$} \\
\hline \multirow[t]{2}{*}{ Cancer } & 2.52 & 1.29 & 1.23 & 0.13 \\
\hline & {$[0.40,15.76]$} & {$[0.22,7.46]$} & {$[0.14,11.19]$} & {$[0.01,2.56]$} \\
\hline \multirow[t]{2}{*}{ Smoker/Past Smoker } & $0.20 *$ & 1.37 & 1.17 & 0.62 \\
\hline & {$[0.04,0.95]$} & {$[0.35,5.41]$} & {$[0.15,9.32]$} & {$[0.10,4.01]$} \\
\hline \multirow[t]{2}{*}{ Cough on presentation } & 0.26 & 0.67 & 0.63 & 3.98 \\
\hline & {$[0.06,1.12]$} & {$[0.18,2.45]$} & {$[0.10,3.89]$} & {$[0.62,25.69]$} \\
\hline \multirow[t]{2}{*}{ Dyspnea on presentation } & 0.60 & 2.74 & $8.93^{\star \star}$ & 3.80 \\
\hline & {$[0.18,2.05]$} & {$[0.77,9.67]$} & {$[1.71,46.58]$} & {$[0.46,31.49]$} \\
\hline \multirow[t]{2}{*}{ Fatigue on presentation } & 0.74 & 1.29 & 1.89 & 3.40 \\
\hline & {$[0.11,5.09]$} & {$[0.25,6.70]$} & {$[0.26,13.72]$} & {$[0.30,38.40]$} \\
\hline \multirow[t]{2}{*}{$\mathrm{SaO} 2$ on presentation } & $1.16^{\star}$ & 0.91 & 0.93 & 0.93 \\
\hline & {$[1.03,1.31]$} & {$[0.81,1.03]$} & {$[0.78,1.11]$} & {$[0.83,1.05]$} \\
\hline \multirow[t]{2}{*}{ Respiratory support } & 0.58 & 1.19 & 4.03 & 2.33 \\
\hline & {$[0.08,4.11]$} & {$[0.12,11.66]$} & {$[0.28,58.47]$} & {$[0.11,48.09]$} \\
\hline \multirow[t]{2}{*}{ Antibiotics treatment } & 2.33 & 0.20 & 2.74 & 2.11 \\
\hline & {$[0.61,8.95]$} & {$[0.03,1.18]$} & {$[0.59,12.82]$} & {$[0.50,8.93]$} \\
\hline \multirow[t]{2}{*}{ Remdesivir treatment } & $6.56^{\star}$ & 0.39 & 2.94 & 0.81 \\
\hline & {$[1.21,35.51]$} & {$[0.06,2.33]$} & {$[0.39,22.38]$} & {$[0.11,6.27]$} \\
\hline \multicolumn{5}{|l|}{${ }^{*} p<0.05$} \\
\hline $\mathrm{SaO} 2=$ oxygen saturation & room air & & & \\
\hline
\end{tabular}




\begin{tabular}{|lllll|}
\hline & Residual Symptoms & Reduced FVC & Abnormal CXR & Abnormal CT \\
\hline \begin{tabular}{lllll|} 
Dexamethasone \\
treatment
\end{tabular} & 0.45 & 3.37 & 0.18 & 0.04 \\
& {$[0.05,4.40]$} & {$[0.25,53.77]$} & {$[0.01,5.39]$} & {$[0.00,1.12]$} \\
\hline Anticoagulation treatment & $7.43^{*}$ & 0.13 & 1.02 & 10.02 \\
& {$[1.01,54.47]$} & {$[0.01,1.33]$} & $0.08,13.33]$ & {$[0.84,119.12]$} \\
\hline${ }^{*}<0.05$ & & & & \\
\hline SaO2 = oxygen saturation on room air & & & \\
\hline
\end{tabular}

Respiratory outcomes included reduced FVC, abnormal CXR and abnormal CT findings at follow up. Older age, male gender, and dyspnea during acute illness were associated with a higher likelihood of abnormal CXR at follow up $(\mathrm{OR}=1.11,6.12$, and 8.93 , respectively). Older age was associated with a higher likelihood of abnormal CT findings at follow up $(O R=1.11)$. Treatment of patients with anticoagulation had a higher probability to predict abnormal CT findings in comparison with patients without this treatment, but without statistical significance.

\section{Discussion}

In this study, we found that survivors of COVID-19 continue to suffer from reduced quality of life and residual symptoms 12 weeks after acute disease, particularly shortness of breath and fatigue. Prolonged symptoms are present regardless of initial disease severity. Patients with severe initial COVID-19 exhibit more impairment of lung function and imaging findings at three months than patients with mild or moderate disease.

To our knowledge, only a few studies with a small number of subjects have compared the frequency of prolonged symptoms in COVID-19 survivors with different disease severity ${ }^{14,21}$. Symptoms following acute COVID-19 are common and have been well documented. Our findings are consistent with previous studies that report that fatigue and shortness of breath are the most common residual symptoms in survivors of COVID-19 11-15,18-22. Persistent symptoms are extremely common, even in patients with mild acute disease. This group accounts for the large majority of COVID-19 infected patients, most of whom are outpatients. Abdallah et al. showed persistent fatigue and exertional breathlessness in $81.6 \%$ of nonhospitalized COVID-19 survivors, but without impairment of lung function or cardiopulmonary exercise test $^{21}$. Logue et al. reported persistent symptoms in $65.3 \%$ of 150 outpatients with mild disease ${ }^{11}$. Other studies show similar findings $12,14,15,22$. Our study and others clearly demonstrate that even patients who suffered from mild acute COVID-19 disease frequently develop a wide range of residual symptoms with ongoing disability and impaired quality of life, but without abnormalities in pulmonary function tests or imaging studies. 
In contrast to mild disease, survivors of severe COVID-19 had significant pulmonary sequalae, with evidence of reduced FEV1, FVC and TLC, and abnormal chest imaging (CXR or CT) at 3 months of follow up. Our findings are consistent with previous reports that focused on the evaluation of severe COVID-19 survivors $18,19,27,28$. A reduction in diffusion capacity is the most commonly reported physiologic impairment in these patients ${ }^{5}$ and our findings also support that, although in our study the difference in DLCO did not reach statistical significance. In accordance with previous reports, restrictive impairment of lung function is the major pattern, as demonstrated in our study.

Our findings highlight the fact that inpatients and survivors of severe COVID-19 should be the main focus of follow up and may benefit from a systematic approach to evaluate for the presence of residual pulmonary injury. Given the significant morbidity that SARS-CoV-2 continue to cause, more survivors of severe COVID-19 are expected to present to post-COVID outpatient clinics or continue to be managed into medical departments. Thus, there is a need for early identification of those patients, rigorous follow up protocol and early treatment of their pulmonary sequalae. More accurate predictors for early identifications such as molecular biomarkers may help in identifications of these patients and preventing persistent pulmonary damage.

The management of post-COVID is still an area of active research, and treatment of a subset of pulmonary residual damage with corticosteroids may be beneficial. Our study was not designed to address this question, but findings show that majority of the patients with post-COVID residual pulmonary damage had inflammatory lung disease consistent with organizing pneumonia or ground glass opacities on CT scan. A majority of our severe patient group were treated with corticosteroids. One small UK cohort demonstrated significant symptomatic and radiological improvement of similar patients with corticosteroids ${ }^{29}$. Thus, corticosteroids should be considered as a treatment option for a subset of post-COVID patients with inflammatory pulmonary complications.

Our study has several limitations. This is a single center cohort analysis, follow up was evaluated within 12 weeks from initial disease, and longer-term data is not yet available. Patient selection for our cohort is subject to bias as we included "all comers" to the hospital post-COVID clinic. There are many factors which may have influenced which patients arrived for follow-up, with symptomatic patients more likely to come for follow-up visits than those who have no residual manifestations. We are thus likely to have overestimated poor health and residual symptoms in our cohort, particularly in the mild patient group. Not all patients agreed to perform all follow-up tests, such as $\mathrm{x}$-ray and pulmonary function tests, but this is unlikely to have significantly influenced overall findings.

\section{Conclusion}

We interpret our study findings in the following way: post-COVID-19 symptoms should be considered as following two different trajectories, which certainly overlap and often coexist but require different approaches for treatment and follow-up. Firstly, non-specific ill-defined symptoms are extremely common and seem to be as frequent following mild as following more severe acute-COVID disease. This group of 
patients is no-doubt very diverse and is likely to include both inpatients and outpatients. The pathophysiological basis for these symptoms is unclear, as is treatment. For many of these patients, health advice and reassurance may suffice. The second trajectory includes patients with evidence of endorgan injury, particularly respiratory impairment, but also cardiac, neurological and other pathologies. Extensive laboratory evaluation such as full pulmonary function tests, imaging, and echocardiography etc., should be limited to the latter group of patients.

\section{Abbreviations}

COVID: Coronavirus induces disease, SARS-Cov-2: Severe acute respiratory syndrome coronavirus 2, FEV1: Forced expiratory volume in second 1 ,FVC: Forced vital capacity, TLC: Total lung capacity , RV: residual volume, Dlco: Diffusion capacity of carbon monoxide , CT: computerized tomography , PFT: pulmonary function tests, Sa02: oxygen saturation, SGRQ: Saint George respiratory questionnaire, CXR: Chest $x$ ray, HFNC: High flow nasal cannula

\section{Declarations}

\section{Ethics approval}

The study protocol was approved by the ethics committee of Hadassah - Hebrew University Medical Center (number HMO-0294-20) and informed consent was obtained from all participants in the study.

\section{Consent for publication}

informed consent was obtained from all participants in the study

\section{Availability of data and material}

the data will not be shared to reserve patient privacy and not signing them on this issue as a part of the informed consent

\section{Funding}

No financial grants or funding sources

\section{Competing interests}

The authors declare that they have no competing interests.

\section{Authors' contributions}

F.D. Is the guarantor of the article and takes responsibility for the integrity of the work, including data and analysis of the article. F.D. and N.B. conceptualized the research aims and planned the analyses. A.A.R, K.K., D.P, U.L., Z.F., R.K. and P.G.C. collected the clinical data. F.D. participated in processing data and 
performing the statistical analysis. R.K. and N.B. wrote part of the manuscript. F.D. wrote the manuscript. All authors contributed to the acquisition, analysis, or interpretation of data. All authors revised the report and approved the final version before submission

\section{Acknowledgements}

Not applicable

\section{References}

1. Zhu N, Zhang D, Wang W, et al. A novel coronavirus from patients with pneumonia in China, 2019. New England Journal of Medicine. Published online 2020. doi:10.1056/NEJMoa2001017.

2. Guan W, Ni Z, Hu Y, et al. Clinical Characteristics of Coronavirus Disease 2019 in China. New England Journal of Medicine Published online. 2020. doi:10.1056/nejmoa2002032.

3. NIH. Coronavirus Disease 2019 (COVID-19) Treatment Guidelines. Disponible en: https://covid19treatmentguidelines.nih.gov/. Nih. 2020;2019:130.

4. Gupta A, Madhavan M v., Sehgal K, et al. Extrapulmonary manifestations of COVID-19. Nat Med. 2020;26(7). doi:10.1038/s41591-020-0968-3.

5. Nalbandian A, Sehgal K, Gupta A, et al. Post-acute COVID-19 syndrome. Nature Medicine Published online April 1, 2021. doi:10.1038/s41591-021-01283-z.

6. Greenhalgh T, Knight M, A'Court C, Buxton M, Husain L. Management of post-acute covid-19 in primary care. The BMJ. 2020;370. doi:10.1136/bmj.m3026.

7. British Thoracic Society Guidance on Respiratory Follow Up of Patients with a Clinico-Radiological Diagnosis of COVID-19 Pneumonia.

8. Your Responsibility.; 2021. https://www.nice.org.uk/terms-and-.

9. Carfi A, Bernabei R, Landi F. Persistent Symptoms in Patients after Acute COVID-19. JAMA - Journal of the American Medical Association. Published online 2020. doi:10.1001/jama.2020.12603.

10. Mandal S, Barnett J, Brill SE, et al. "Long-COVID": a cross-sectional study of persisting symptoms, biomarker and imaging abnormalities following hospitalisation for COVID-19. doi:10.1136/thoraxjnl2020-215818.

11. Logue JK, Franko NM, McCulloch DJ, et al. Sequelae in Adults at 6 Months after COVID-19 Infection. JAMA Network Open. 2021;4(2). doi:10.1001/jamanetworkopen.2021.0830.

12. Havervall S, Rosell A, Phillipson M, et al. Symptoms and Functional Impairment Assessed 8 Months After Mild COVID-19 Among Health Care Workers. JAMA Published online April 7, 2021. doi:10.1001/jama.2021.5612.

13. Morin L, Savale L, Pham T, et al. Four-Month Clinical Status of a Cohort of Patients after Hospitalization for COVID-19. JAMA - Journal of the American Medical Association. Published online 2021. doi:10.1001/jama.2021.3331. 
14. Townsend L, Dowds J, O'Brien K, et al. Persistent Poor Health Post-COVID-19 Is Not Associated with Respiratory Complications or Initial Disease Severity. Annals of the American Thoracic Society. Published online January 8, 2021. doi:10.1513/annalsats.202009-1175oc.

15. Davis HE, Assaf GS, McCorkell L, et al. Characterizing long COVID in an international cohort: 7 months of symptoms and their impact. medRxiv Published online December 27, 2020. doi:10.1101/2020.12.24.20248802.

16. Zhao $Y$, Shang $Y$ min, Song $W$ bin, et al. Follow-up study of the pulmonary function and related physiological characteristics of COVID-19 survivors three months after recovery. EClinicalMedicine. Published online 2020. doi:10.1016/j.eclinm.2020.100463.

17. van der Sar - van der Brugge S, Talman S, Boonman - de Winter LJM, et al. Pulmonary function and health-related quality of life after COVID-19 pneumonia. Respiratory Medicine. Published online 2021. doi:10.1016/j.rmed.2020.106272.

18. Smet J, Stylemans D, Hanon S, Verbanck S, Vanderhelst E, Ilsen B. Clinical status and lung function 10 weeks after severe SARS-CoV-2 infection. Respir Med. 2021;176.

doi:10.1016/j.rmed.2020.106276.

19. Daher A, Balfanz P, Cornelissen C, et al. Follow up of patients with severe coronavirus disease 2019 (COVID-19): Pulmonary and extrapulmonary disease sequelae. Respir Med. 2020;174. doi:10.1016/j.rmed.2020.106197.

20. Huang C, Huang L, Wang Y, et al. 6-month consequences of COVID-19 in patients discharged from hospital: a cohort study. The Lancet. Published online 2021. doi:10.1016/S0140-6736(20)32656-8.

21. Abdallah SJ, Voduc N, Corrales-Medina VF, et al. Symptoms, Pulmonary Function and Functional Capacity Four Months after COVID-19. Annals of the American Thoracic Society. Published online April 19, 2021. doi:10.1513/AnnalsATS.202012-1489RL.

22. Trinkmann F, Müller $M$, Reif $A$, et al. Residual symptoms and lower lung function in patients recovering from SARS-CoV-2 infection. Eur Respir J. 2021;57(2). doi:10.1183/13993003.03002-2020.

23. St. George's Respiratory Questionnaire (SGRQ). Accessed April 29. 2021. https://www.thoracic.org/members/assemblies/assemblies/srn/questionaires/sgrq.php.

24. Santus P, Tursi F, Croce G, et al. Changes in Quality of Life and Dyspnoea after Hospitalization in COVID-19 Patients Discharged at Home.

25. Graham BL, Steenbruggen I, Barjaktarevic IZ, et al. Standardization of spirometry 2019 update an official American Thoracic Society and European Respiratory Society technical statement. American Journal of Respiratory and Critical Care Medicine. Published online 2019. doi:10.1164/rccm.201908$1590 \mathrm{ST}$.

26. Pellegrino R, Viegi G, Brusasco V, et al. "ATS/ERS task force: standardisation of lung function testing." Interpretative strategies for lung function tests. European Respiratory Journal. Published online 2005. doi:10.3174/ajnr.A1614.

27. van der Sar - van der Brugge S, Talman S, Boonman - de Winter LJM, et al. Pulmonary function and health-related quality of life after COVID-19 pneumonia. Respir Med. 2021;176. 
doi:10.1016/j.rmed.2020.106272.

28. van Gassel RJJ, Bels JLM, Raafs A, et al. High prevalence of pulmonary sequelae at 3 months after hospital discharge in mechanically ventilated survivors of COVID-19. Am J Respir Crit Care Med. 2021;203(3):371-4. doi:10.1164/rccm.202010-3823LE.

29. Myall KJ, Mukherjee B, Castanheira AM, et al. Persistent Post-COVID-19 Inflammatory Interstitial Lung Disease: An Observational Study of Corticosteroid Treatment. Annals of the American Thoracic Society. Published online January 12, 2021. doi:10.1513/annalsats.202008-1002oc.

\section{Figures}

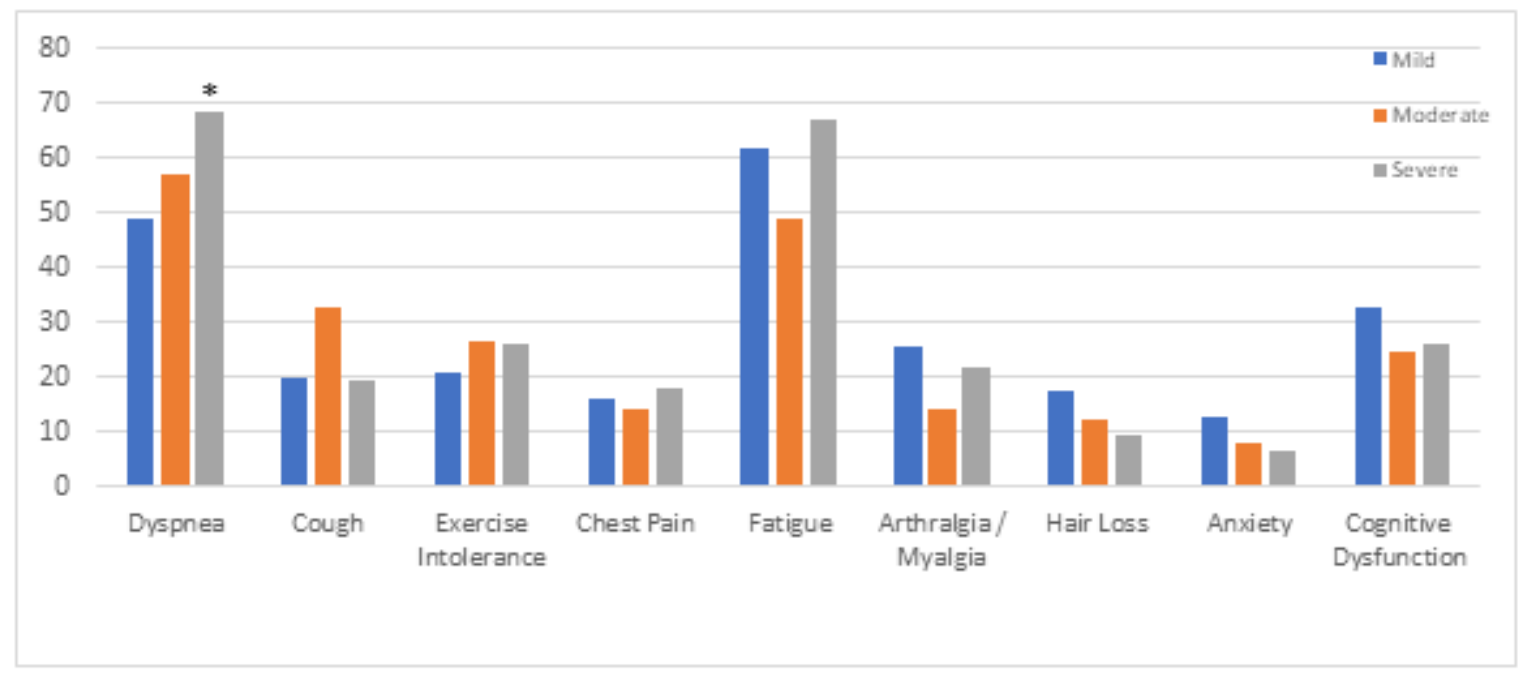

a

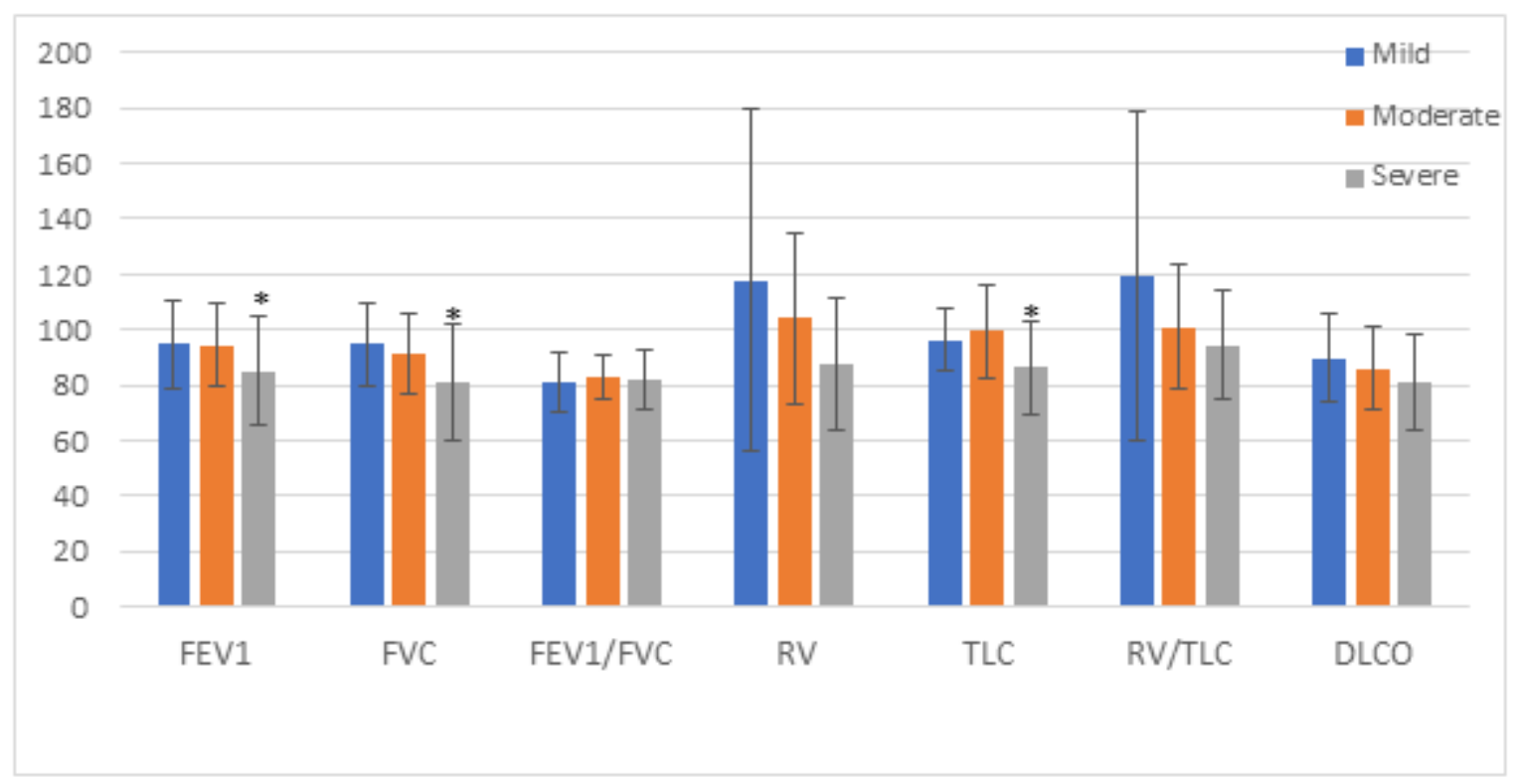

Figure 1 
a: Residual symptoms frequency comparison between patients according to initial diseases severity: Mild, moderate and severe COVID-19. * $\mathrm{P}<0.05$ b: pulmonary function tests parameters on follow up according to initial diseases severity: Mild, moderate and severe COVID-19.

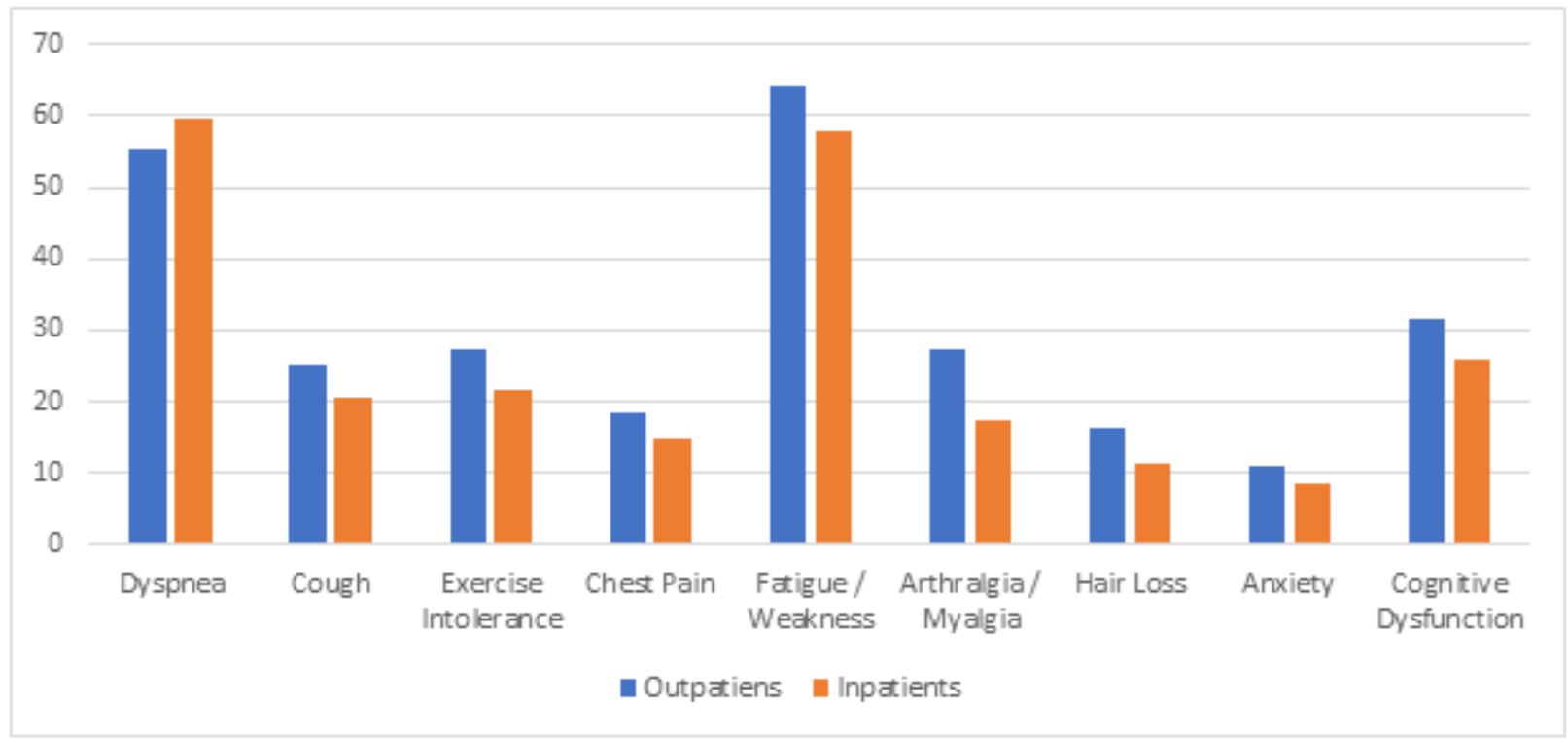

a

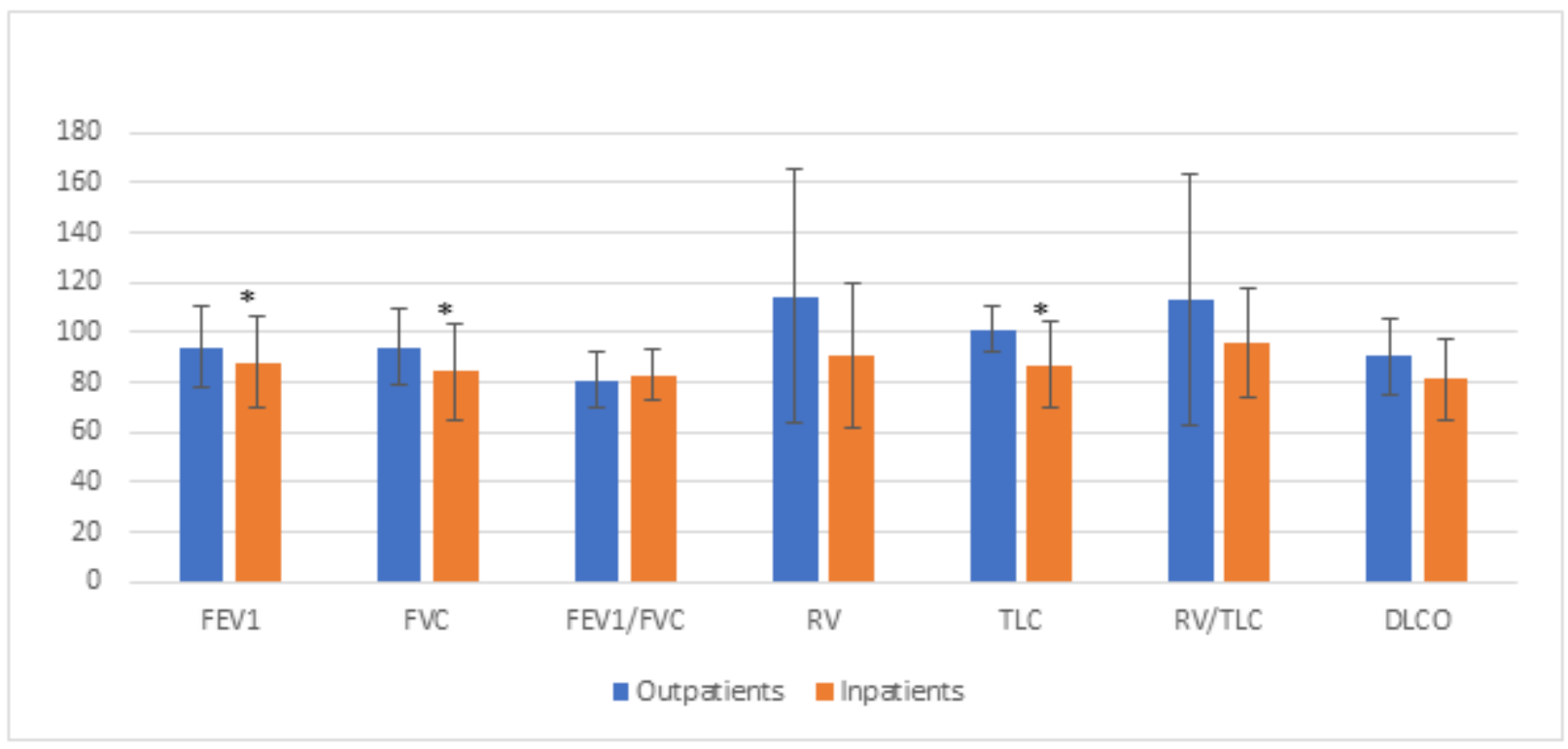

b

Figure 2

a: Residual symptoms frequency comparison between Inpatients and outpatients. * $-\mathrm{P}<0.05 \mathrm{~b}$ : pulmonary function tests parameters on follow up compared between inpatients and outpatients. * $\mathrm{P}$ $<0.05$ 